\title{
SCHWERPUNKT: NATUR UND NATURALISIERUNG BEI SPINOZA
}

Die Diskussion und Rezeption von Spinozas Philosophie war in den letzten Jahrzehnten deutlich von sehr unterschiedlichen theoretischen Interessen geprägt, vor deren Hintergrund seine Werke jeweils interpretiert wurden: Seit den 60er Jahren thematisierte die Analytische Philosophie insbesondere den logischen und argumentativen Status spinozanischer Gedankengänge. In Frankreich wurde Spinoza in der zweiten Hälfte der 60er Jahre zu einem zentralen Referenzpunkt der poststrukturalistischen Auseinandersetzung mit dem dort wirkmächtigen cartesischen Erbe. In Italien und besonders in den Niederlanden wurde Spinoza überwiegend in historisch-philologischen Forschungszusammenhängen rezipiert. In Deutschland hatte der Nationalsozialismus der bis 1933 regen Spinoza-Forschung zunächst ein Ende gesetzt, sodass sich eine deutschsprachige Diskussion nach einzelnen Anfängen in den 60er und 70er Jahren erst in den letzten Jahrzehnten wieder zu entwickeln begann. Entstanden sind dabei eine Reihe von Monographien und Einzelstudien, die sich intensiv mit der Spinoza-Rezeption auseinandersetzten oder auf das Ganze von Spinozas Philosophie abzielten, dabei aber nur wenig aufeinander Bezug nahmen.

In jüngster Zeit hat sich die Wahrnehmung von Spinozas Philosophie in Deutschland wie auch international deutlich gewandelt. Angeregt durch Überlegungen von Autoren wie Jonathan Israel und Antonio Damasio, ist Spinoza zu einem Verbündeten in vielen systematisch geführten Sachdiskussionen sowohl im Bereich der theoretischen als auch in der praktischen Philosophie geworden. In der Folge dieser Entwicklung wird Spinoza auch in der deutschsprachigen Forschung zu einem Gesprächspartner, sei es für die politische Theorie, sei es für die Auseinandersetzung mit der therapeutischen Funktion von Philosophie, sei es, dass er als ein Referenzpunkt für die Naturalismus-Debatte fungiert. Der Rückgang auf Spinoza erlaubt eine kritische Auseinandersetzung mit dem Naturbegriff, der viele, scheinbar unproblematische Topoi aufbricht, wie sie etwa in der Rede von der kausalen Determiniertheit der Natur oder der unreflektierten Entgegensetzung von Natur und Kultur enthalten sind. In historisch informierten und analytisch gründlichen Lesarten werden Grundbegriffe von Spinozas Philosophie problematisiert und begrifflich weiterentwickelt und gewohnte Etikettierungen - wie die des Antidualismus, des Naturalismus seiner Epistemologie und Politik sowie die vom Pantheismus seines Ansatzes - erneut der kritischen Reflexion ausgesetzt.

Die folgenden fünf Beiträge gehen auf Vorträge zurück, die im Oktober 2008 im Rahmen der 10. Tagung der Spinoza-Gesellschaft in Marburg gehalten wurden. Diese Auswahl bildet weder das Programm der weit über Spinoza hinausgehenden Tagung ab, noch repräsentiert sie den Stand der Spinoza-Forschung insgesamt. Die Herausgeberinnen hielten die abgedruckten Beiträge vielmehr für geeignet, einen Eindruck von der oben skizzierten Entwicklung innerhalb der deutschen Spinoza-Diskussion zu vermitteln. Die Texte sollen sichtbar machen, auf welche Weise insbesondere jüngere Wissenschaftlerinnen und Wissenschaftler, die mitunter von durchaus unterschiedlichen methodischen Grundlagen ausgehen, die Frage nach Spinozas Naturalismus bearbeiten. 
So warnt etwa Han van Ruler in seinem Beitrag Spinozas doppelter Dualismus davor, Spinozas Philosophie vorschnell dadurch in einem naturalistischen Sinne zu interpretieren, dass man seinen Begriff von Kausalität in eine unmittelbare Verbindung mit dem der heutigen Psychologie oder Physik bringt. Spinozas Monismus und Parallelismus sind vielmehr aus dem Anliegen entstanden, eine theoretische Antwort auf die Paradoxa des Cartesianismus zu geben. Die Ethik stellt so gesehen einen heroischen Versuch dar, im Rahmen des neu entstandenen mechanistischen Denkens den Bedingungen einer Moralphilosophie gerecht zu werden. In einer philosophiehistorischen Analyse arbeitet van Ruler heraus, dass sich bei Spinoza nicht nur Restbestände des cartesischen Dualismus' nachweisen lassen, sondern dass man seinen Dualismus der Attribute sogar als eine Radikalisierung des cartesischen Dualismus' lesen muss. In Spinozas Ontologie der Verursachung erkennt van Ruler eine weitere Form des Dualismus, die seiner Ansicht nach in Kontinuität mit der platonisch-aristotelischen Metaphysik steht. Spinozas Akzeptanz dieser beiden Dualismen hat aber nicht nur Konsequenzen für die moralphilosophischen Lehren in seiner Ethik, sondern lässt auch den Versuch, seine Theorie in Übereinstimmung mit heutigen Formen des naturalistischen Denkens zu bringen, fragwürdig werden.

Ursula Renz stellt sich in ihrem Aufsatz Spinozas Erkenntnistheorie: Eine naturalistische Epistemologie? die Frage, inwiefern die auf den ersten Blick offenkundigen Naturalismen der verschiedenen Theoriestücke der Ethik mit gegenwärtigen Auffassungen von Naturalismus zu vergleichen sind. Zwar kann man ihrer Ansicht nach Spinozas Geistkonzeption insofern naturalistisch nennen, als Bewusstseinsphänomene des subjektiven Erlebens darin als vollständig erklärbar bestimmt werden. Mindestens ebenso entscheidend ist ihrer Ansicht nach aber, dass sich Spinoza radikal von jeglichem Mythos des Gegebenen verabschiedet hat. Im Rekurs auf Spinozas Theorie der „,imaginatio“, sein Konzept der „notiones communes“ und seine Rede von der ,idea vera“ zeigt Renz ferner, dass seine Erkenntnistheorie Erkenntnis als einen normativen Begriff bestimmt und sich mit der Überlegung befasst, was wir eigentlich voraussetzen müssen, um an der Möglichkeit von Erkenntnis festhalten zu können. Nimmt man dieses Verständnis von Spinozas Begriff der Erkenntnis zum Maßstab, so muss man zum Schluss kommen, dass er in seiner Erkenntnistheorie primär Transzendentalphilosoph und nicht Naturalist ist.

Die Frage nach der Natur des Menschen stellt sich bei Spinoza aber nicht nur im Zusammenhang der theoretischen, sondern auch im Zusammenhang seiner politischen Philosophie. Martin Saar hebt in seinem Text Politik der Natur. Spinozas Begriff der Regierung zunächst die integrative Leistung von Spinozas ,politischem Naturalismus“ hervor. Spinoza denkt den politischen Menschen gleichzeitig als naturales Wesen und begründet damit das Erfordernis, den Menschen auch als ein solches zu behandeln und seiner Einbindung in ein naturgesetzlich strukturiertes Ganzes Rechnung zu tragen. Dieser Zugang hat tiefgreifende Folgen für die Art und Weise, wie und mit welchen Mitteln Spinoza über Politik nachdenkt, und führt zu einer Vorstellung vom Regieren, die ihrerseits diese beiden Aspekte einschließt. Saar arbeitet heraus, dass Spinozas Begriff der Regierung zugleich ein juridischer, ein praktischer und ein reflexiver Begriff ist und in dieser scheinbar problematischen Mehrdeutigkeit gerade Spinozas originelle philosophische These über den Zusammenhang dieser drei Dimensionen liegt. Politische Steuerung oder Regierung muss entsprechend der Dimensionierung des Begriffs als ein Zusammenspiel unterschiedlicher effektiver Kräfte gefasst werden. Spinoza kann daher als frühmoderner Theoretiker einer „Politik der Natur“ und als Vorläufer einer modernen „biopolitischen“ Konzeption von Politik gelesen werden, auch wenn seine Theorie eine aktive, dynamische „Politik des Lebens“ nur vorzeichnet, aber nicht selbst beschreitet. 
Johannes Haag erkennt in Spinozas Ideentheorie eines der wichtigsten und gleichzeitig umstrittensten Lehrstücke seiner Ethik. In seinem Aufsatz Spinoza über die Intentionalität geistiger Zustände schlägt er eine Neuinterpretation dieser Theorie vor. Sie soll es erlauben, Spinoza gerade als Wegbereiter einer naturalisierten Theorie der Intentionalität zu begreifen. Ausgangspunkt von Haags Überlegungen ist die von Spinoza geforderte prinzipielle Unterscheidung zwischen göttlichem, unendlichem und menschlichem, endlichem Verstand. Um dieser Unterscheidung gerecht zu werden, differenziert Haag zunächst zwischen zwei Repräsentationsrelationen - einer Relation, die Ideen zu vollwertigen intentionalen Zuständen macht, und einer nicht-intentionalen Repräsentationsrelation. Er macht im Anschluss aber deutlich, dass sich beide doch wieder auf eine grundlegende Anzeigerelation reduzieren lassen, jedoch ohne dass der Ideenbegriff dadurch in die oft behauptete Ambiguität gerät. Während die Differenzierung der Repräsentationsrelation wichtige epistemologische Konsequenzen hat, versucht Haag zu zeigen, dass mit der Möglichkeit, diese Relationen auf eine zu Grunde liegende Anzeigerelation zu reduzieren, die Naturalisierung der Intentionalität geistiger Zustände gelingt. Auch wenn Haag zu dem Ergebnis gelangt, dass Spinozas Idee der semantischen Abbildung nicht zur Grundlage eines epistemischen Ideals taugt, bleibt sie seiner Ansicht nach doch von systematischem Interesse für eine Theorie naturalisierter Intentionalität. Diesen Gedanken skizziert Haag am Ende seines Beitrages anhand einer knappen kontrastierenden Gegenüberstellung mit der zeitgenössischen Abbildtheorie von Wilfrid Sellars.

Ausgesprochen kritisch beurteilt Winfried Schröder die Möglichkeit, systematisch an die Philosophie Spinozas anzuschließen. Seine Untersuchung nimmt zunächst den Ausgang von dem Begriff des ,Pantheismus', mit dem die Metaphysik Spinozas nach wie vor häufig betitelt wird. In einem ersten Schritt klärt Schröder über die problematische begriffliche Struktur dieses Begriffs auf und erweist ihn als ein Oxymoron und ein ,unseriöses Spiel mit Worten'. Bereits die Diskussion der Tradition hat darauf aufmerksam gemacht, dass mit dem Begriff des Pantheismus die Grenzen möglicher Revisionen des Gottesbegriffs überschritten werden. Seit der Aufklärung steht er nun für eigentümliche weltanschauliche Mischgebilde, die philosophische mit religiösen Versatzstücken kombinieren. Vor allem seit der Spinoza-Rezeption im Gefolge von Lessings Bekenntnis zu Spinoza hatte diese Art von Weltanschauung bis weit ins 19. Jahrhundert eine bemerkenswerte Konjunktur. Dabei kam es, wie Schröder aufzeigt, begrifflich zur Synonymie von ,Spinozismus' und ,Pantheismus' und der Sache nach zur Betonung der religiösen oder quasireligiösen Elemente in Spinozas Metaphysik. Diese Entwicklung wertet Schröder aber nicht als Effekt eines unglücklichen Zufalls oder eines grundsätzliches Missverständnisses der Rezeptionsgeschichte von Spinozas Philosophie. Er nimmt sie vielmehr zum Anlass, die Metaphysik der Ethica, insbesondere die Lehre vom ,amor dei intellectualis“, genauer daraufhin zu befragen, ob sie der Formation einer solchen Weltanschauung nicht tatsächlich selbst Vorschub geleistet hat. In seiner Analyse gelangt Schröder schließlich zu dem Ergebnis, dass Spinozas Deus sive natura selbst schon ein solches problematisches philosophisch-religiöses Hybridgebilde ist und daher Anschlüsse heutigen Philosophierens an die Metaphysik Spinozas durchaus kritisch zu hinterfragen seien. Ein säkularistisch halbierter Spinoza ist aus Schröders Sicht letztlich unverständlich, weshalb er die Möglichkeit, systematisch an Spinoza anzuschließen, grundsätzlich in Frage stellt. 